\title{
Cognitive Radio Application for Smart Grid
}

\author{
Vasudev Dehalwar ${ }^{\mathrm{a}}$, Mohan Kolhe ${ }^{\mathrm{b}, *}$, Sunita Kolhe ${ }^{\mathrm{b}}$ \\ ${ }^{a}$ Department of Computer Science and Engineering, MA National Institute of Technology, Bhopal, India \\ ${ }^{b}$ Faculty of Engineering and Science, University of Agder, Grimstad 4879, Norway
}

\begin{abstract}
The communication infrastructure between energy generation, transmission, distribution and utilization will require multi-way communications, interoperability between the advanced and existing system, and end-to-end reliable and secure communications with low-latencies. Revolutionary communication architecture is required for effective operation and control of smart grid, and cognitive radio based communication architecture can provide a unique solution. By leveraging cognitive radio technology, the suggested communications infrastructure promises to utilize potentially all available spectrum resources efficiently in the smart grid. The radio agility allows the smart grid devices to sense the unused spectrum opportunities in the surroundings and utilize them subject to interference constraints. Dynamic spectrum access enabled by cognitive radio technology can be adopted by the smart grid to exploit the underutilized frequencies in an opportunistic manner. As a result, the flexibility, efficiency, and reliability can be significantly enhanced in a cognitive radio based smart grid network.
\end{abstract}

Keywords: Cognitive radio, smart grid, communication

\section{Introduction}

Traditionally, electricity networks were built for transferring electrical energy generated by highcapacity, centrally placed power production units. However, the electricity industry is now on the edge of making the transformation from a centralized producer controlled network to one that is less centralized and more consumer-interactive. The move to a smarter electricity network promises to change not only the electricity industry's business model but also to accommodate connection of widely distributed, renewable and distributed energy generators across the power system network [1], [2]. A future smart grid power system network will serve as a dynamic network for multi-directional energy flows, linking widely distributed small capacity renewable energy systems at consumer level (distribution network) and centralized higher-capacity power generators, facilitating active participation of customer choice for energy production/source and demand management, and providing real-time multi-direction information on the performance and optimal operation of the power system network [3]. Information technologies will make grid management and control easier on real time and will allow information (communications) between various distributed and centralized generators and consumers. There are many features that are expected to be a part of smart grid, such as advanced metering infrastructure (AMI), real time demandside management (DSM) with respect to real time energy price, fault-tolerance, self-healing, etc [4]-[8]. The AMI will use multi directional communication links between the customers and the utilities for many new services such as energy management and control, active and reactive power management, remote meter reading, power quality, detection of unauthorized usage, etc.

Communication network plays a vital role in the management of information and control instructions throughout the smart grid. Fast, reliable and secure communications are the backbone of all the important

\footnotetext{
* Manuscript received July 10, 2012; revised August 15, 2012.

Corresponding author. Tel.: +47 3723 3293; fax: +47 3814 1001; E-mail address: mohan.l.kolhe@uia.no
} 
decisions in smart grid. The current communication capabilities of the existing power system monitoring i.e. 'supervisory control and data acquisition (SCADA)' are having limited functionalities, which will not fulfill the demanding requirements of smart grid [9]. The geographical spread of the smart grid will be very wide; therefore combination of wired and wireless communication mediums will be used to cover entire geographical region. The data traffic generated will be in terra bytes, hence wired communication with fiber optic may be the best choice [10]. However, at the micro-grid level the communication medium may be wired or wireless, depending on the reliability and availability of data. The prominent wireless technologies available, which can be used at micro-grid/HAN level, include IEEE 802.11 (WiFi), ultra wideband (UWB), IEEE 802.15.4 ZigBee, 6LoWPAN, and so forth [11]-[14].

\section{Smart Grid Communication Architecture}

The smart grid information and communications network will be spread over the large geographical area covering all dimensions of different types of power generation, transmission, distribution and utilization. The home area network (HAN) will provide access to in-home appliances, while the neighborhood area network (NAN) will connect local energy nodes to the access points, and the wide area network (WAN) will provide the communication links between the grids and core utility control system through multilayer communication system as depicted in Fig $1[5,6]$.

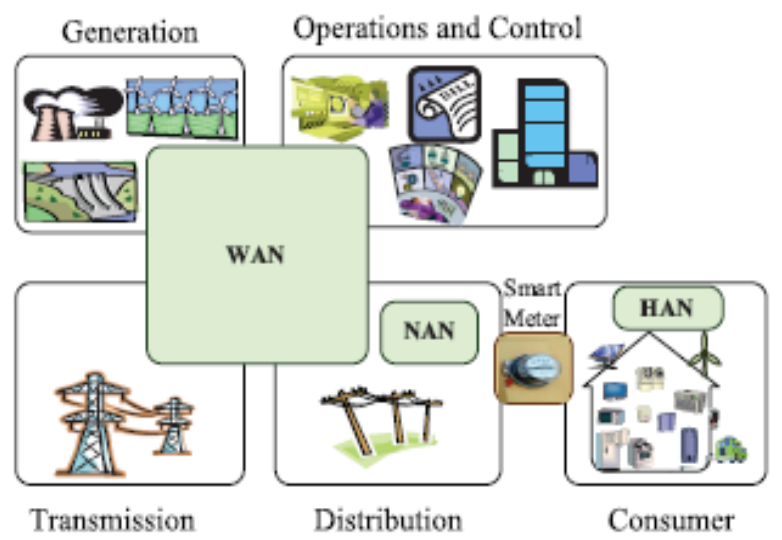

Fig. 1. Multitier network communications architecture [6].

Typically, two types of communication infrastructure will be needed for information flow in a smart grid system. The first flow will be from the energy consumption (at demand level) and the second flow will be between the smart meters and the utility's control and management centers. The first type of data flow can be accomplished through power line communication or carrier communications. The second type of information flow may utilize wired (DSL, SDH, SONET) or wireless technologies (cellular, WIMAX, cognitive radio, etc) or the internet. However, there is no universally acceptable solution for deployment of communication infrastructure in the smart gird. The decision will be based on factors, such as operational costs, the availability of the technology and rural/urban or indoor/outdoor environment, etc. The communication infrastructure between energy generation, transmission, distribution and utilization will require multi-way communications, interoperability between the advanced and existing system, and end-to-end reliable and secure communications with low-latencies [14]-[16]. Moreover, the system should be secure enough to prevent cyber-attacks and provide system stability and reliability with advanced controls. Revolutionary communication architecture is required for effective operation, control and management of smart grid, and cognitive radio based communication architecture can provide a solution. Cognitive radio refers to the wireless systems that are context-aware and capable of reconfiguration based on the surrounding environments and their own properties [17]-[19]. 


\section{Cognitive Radio Application in Smart Grid}

It is important to use the spectrum as efficiently as possible due to increased wireless data traffic. Most of the radio spectrum is currently allotted is through licensing. The existing spectrum is become scare due to increased number of wireless applications and number of users. The spectrum scarcity entails pursuing efficient use of spectrum depending on time, location and frequencies. Spectrum may be classified as licensed, lightly licensed and unlicensed [18]. Licensed spectrum is used for person to person communications (either cellular or land mobile radio used by police and radio taxi services). Lightly licensed spectrum is used by satellite communication and RADAR systems. Unlicensed spectrum of 2.4 $\mathrm{GHz}$ and $5 \mathrm{GHz}$ is used by public also called ISM band.

The research is going on to determine how to use unoccupied or underutilized spectrum for new applications using cognitive radio. The objective of the cognitive radio is to obtain the best available spectrum through cognitive capability and re-configurability. Since most of the spectrum is already assigned, the most important challenge is to share the licensed spectrum without interfering with the transmission of other licensed users as depicted in Fig. 2 [18].

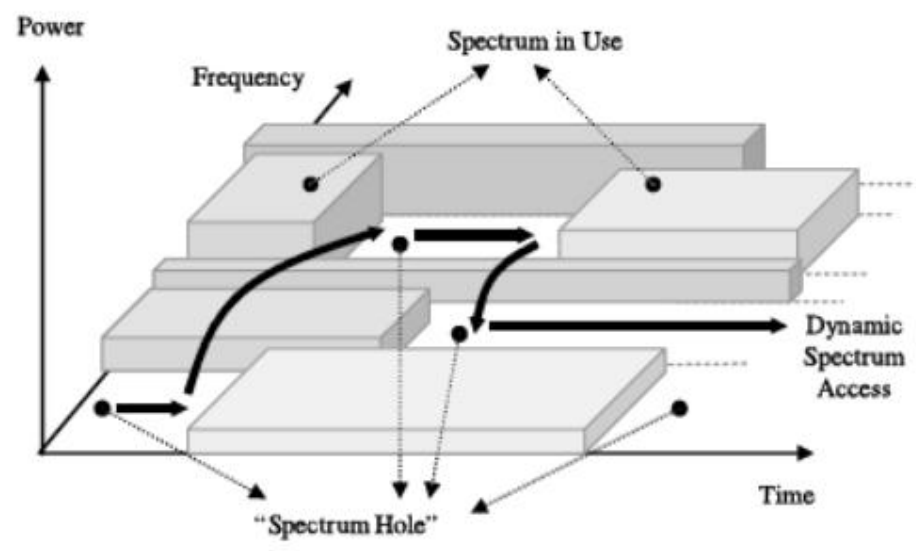

Fig. 2. Spectrum hole concept [18]

The cognitive radio enables the usage of unused spectrum temporally, which is referred to as spectrum hole or white space. If this band is again used by a licensed user, the cognitive radio moves to another spectrum hole or stays in the same band, altering its transmission power level or modulation scheme to avoid interference as depicted in Fig. 2. Dynamic allocation of spectrum is underway for flexible use of traditional spectrum. New technologies are being developed to enable more flexible use of traditional spectrum. The first step involves automating the licensing and database mechanism used in lightly licensed schemes. The transition is taking place from analog TV to digital TV signals. Digital TV reduces the bandwidth requirement of existing broadcast TV. The US regulator FCC has split the TV spectrum across multiple channels, as partially as shown in the Fig. 3 [19].

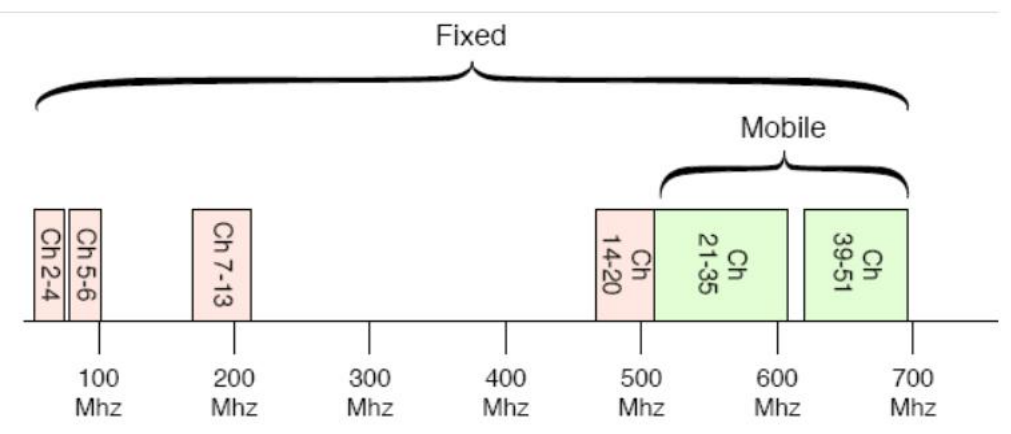

Fig. 3. Spectrum allocation of TV [19] 
Some TV channels are not used, and even where TV channels are used, guard bands are used to reduce the risk of channel interference with one another. These guard bands are unused channels constituting TV White Spaces (TVWS). The FCC divides the TVWS access into two sections in which fixed application or both mobile and fixed applications are allowed. Numbers of prototypes systems are developed showing the TVWS technology is practical. The outcome of the studies says that spectrum sensing technologies can allow the TVWS to be shared by multiple users in the same way as the existing licensed spectrum can be shared by different user. There has been effort in US to devote $30 \mathrm{MHz}$ of TVWS to smart-grid, but due to some reason it has been turned down.

\subsection{Cognitive tasks recognition cycle}

The cognitive process starts with the passive sensing of RF stimuli and culminates with action as depicted in Fig. 4 as proposed by Mitola [17]. There are three on-line cognitive tasks:

(a) Radio-scene analysis, which encompasses the following:

- Estimation of interference temperature of the radio environment;

- Detection of spectrum holes.

(b) Channel identification, which encompasses the following:

- Estimation of channel-state information (CSI);

- Prediction of channel capacity for use by the transmitter

(c) Transmit-power control and dynamic spectrum management.

Tasks (a) and (b) are carried out in the receiver, and task (c) is carried out in the transmitter. Through interaction with the RF environment, these three tasks form a cognitive cycle.

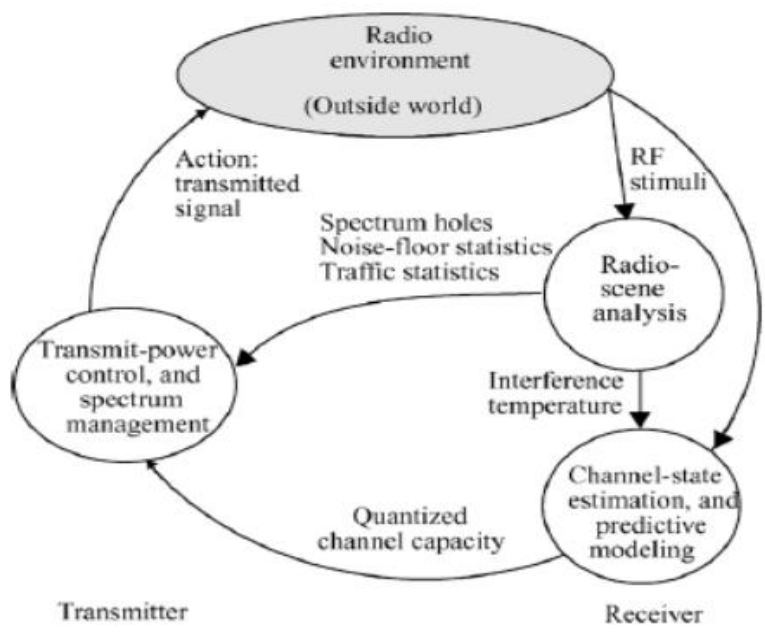

Fig. 4. Basic cognitive cycle [17].

\subsection{Spectrum allocation}

Spectrum allocation can be coordinated through the use of tightly synchronized and highly accurate clocks. If the specific spectrum band is needs by primary user at one location at a specific time, the secondary users of the band could migrate to other band or simply curtail the use of spectrum for short duration [18]. In the same frequency range, there are two coexisting systems: the primary system and the secondary system. The primary system refers to the licensed system with legacy spectrum. This system has exclusive privilege to access the assigned spectrum. The secondary system refers to the unlicensed cognitive system, which can only access the spectrum that is not used by the primary system.

The cognitive radio needs to fulfill the following objectives for the smart grid communication:

- There are several radio systems operating in the $2.4 \mathrm{GHz}$ license-free industrial, scientific, and medical (ISM) frequency band (e.g., Zigbee, Bluetooth, and WiFi). The coexistence of these systems generates significant interference with each other. Apart from that domestic appliances (e.g., microwave ovens) 
may leak strong electromagnetic waves. Therefore, the spectrum in a HAN is becoming crowded and susceptible to interference. It is beneficial to introduce cognitive radio technology in HANs. Based on the parameter-adaptive capacity of cognitive radio, the interference among different radio systems could be considerably reduced by intelligent transmission scheduling and power coordination.

- The generated data shall be in terabytes. It poses a significant challenge as such large bandwidth is not available within existing networks. The usage of cognitive radio in the smart grid should work to improve spectrum utilization and communication capacity to support large-scale data transmissions.

- The smart grid communications architecture shall cover home areas, neighborhood areas, and wide areas. Consequently, it support heterogeneous network with a number of complementary technologies that incorporate intelligent devices/terminals to manage the communications within subarea/substation. For the convergence of the heterogeneous network, smart grid devices, equipped with cognitive radio functionality, should be explored to enable hardware re-configurability and context awareness.

The radio agility allows the smart grid devices to sense the unused spectrum opportunities in the surroundings and utilize them subject to interference constraints. Dynamic spectrum access enabled by cognitive radio technology is adopted by the smart grid to exploit the underutilized frequencies in an opportunistic manner. As a result, the flexibility, efficiency, and reliability are significantly enhanced in a cognitive radio based smart grid network. How to put "cognition and intelligence" into the cognitive radio network will be the focus of the smart grid communication. By leveraging cognitive radio technology, the proposed communications infrastructure promises to utilize potentially all available spectrum resources efficiently in the smart grid.

\section{Conclusion}

Revolutionary communication architecture is required for effective operation and control of smart grid, and cognitive radio based communication architecture can provide a solution. Cognitive radio refers to the wireless systems that are context-aware and capable of reconfiguration based on the surrounding environments and their own properties. In the same frequency range, there are two coexisting systems: the primary system and the secondary system. The primary system refers to the licensed system with legacy spectrum. This system has exclusive privilege to access the assigned spectrum. The secondary system refers to the unlicensed cognitive system, which can only access the spectrum that is not used by the primary system. The primary system refers to the licensed system with legacy spectrum. This system has exclusive privilege to access the assigned spectrum. The secondary system refers to the unlicensed cognitive system, which can only access the spectrum that is not used by the primary system. By leveraging cognitive radio technology, the proposed communications infrastructure promises to utilize potentially all available spectrum resources efficiently in the smart grid.

\section{References}

[1] Güngör VC, Sahin Kocak DT, Ergut S, Buccella C, Cecati C. Smart grid technologies: communication technologies and standards. IEEE Trans. on Industrial Informatics, 2011; 7(4):529-539.

[2] Budka KC, Deshpande JG, Doumi TL, Madden M, Mew T. Communication network architecture and design principles for smart grids. Bell Labs Technical Journal, 2010; 15(2):205-228.

[3] Kolhe M. Smart grid: charting a new energy future: research, development and demonstration. The Electricity Journal, 2012; 25(2): 88-93.

[4] Han Y, Wang J, Zhao Q, Han P. Cognitive information communication network for smart grid. In: Proc. of IEEE International Conference on Information Science and Technology, 2012: 847-850.

[5] Qiu RC, Hu Z, Chen Z, Ranganathan R, Hou S, Zheng G. Cognitive radio network for the smart grid: experimental system architecture, control algorithms, security, and microgrid testbed. IEEE Trans on Smart Grid, 2011; 2(2):724-740.

[6] Ghassemi A, Bavarian S, Lampe L. Cognitive radio for smart grid communications. In: Proc. of First IEEE International Conference on Smart Grid Communications, 2010:297-302.

[7] Yu R, Zhang Y, Gjessing S, Yuen C, Xie S, Guizani M. Cognitive radio based hierarchical communications infrastructure for smart grid. IEEE Network, 2011; 25(5):6-14. 
[8] Dehalwar V, Baghel RK, Kolhe M. Multi-agent based public key infrastructure for smart grid. Presented at: IEEE 7th International Conference on Computer Science \& Education.

[9] Meliopoulos APS, Cokkinides G, Huang R, Farantatos E, Choi S, Lee Y, Yu X. Smart grid technologies for autonomous operation and control. IEEE Trans on Smart Grid, 2011; 2(1):1-10.

[10] Wang J, Ghosh M, Challapali K. Emerging cognitive radio applications: a survey. IEEE Communications Magazine, 2011; 49(3):74-81.

[11] Wang W, Xu Y, Khanna M, A survey on the communication architectures in smart grid. Computer Networks, 2011; 55(15): 3604-3629.

[12] Gao J, Xiao Y, Liu J, Liang W, Chen CLP. A survey of communication/networking in smart grids. Future Generation Computer Systems, 2012; 28(2):391-404.

[13] Li F, Qiao W, Sun H, Wan H, Wang J, Xia Y, Xu Z, Zhang P. Smart transmission grid: vision and framework. IEEE Trans. on Smart Grid, 2010; 1(2):168-177.

[14] Cecati C, Mokryani G, Piccolo A, Siano P. An overview on the smart grid concept. In: Proc. of 36th Annual Conference on IEEE Industrial Electronics Society, 2010: 3322-3327.

[15] Zhang Y, Yu R, Nekovee M, et al. Cognitive machine-to-machine communications: visions and potentials for the smart grid. IEEE Network, 2012; 26(3):6-13.

[16] Fadlullah ZM, Fouda MM, Kato N, et al. Toward intelligent machine-to-machine communications in smart grid. IEEE Communications Magazine, 2011; 49(4):60-65.

[17] Haykin S. Cognitive radio: brain-empowered wireless communications. IEEE Journal on Selected Areas in Communications, 2005; 23(2): 201-220.

[18] Akyildiz IF, Lee W-Y, Vuran MC, Mohanty S. NeXt generation/dynamic spectrum access/cognitive radio wireless networks: a survey. Computer Networks, 2006; 50(13):2127-2159.

[19] Grunwald D. How new technologies can turn a spectrum crisis into a spectrum opportunity. University of Colorado. (Feb 2011). [Online]. Available: http://systems.cs.colorado.edu/wp-content/uploads/2011/02/Spectrum-Crisis-SpectrumOpportunity.pdf 